\title{
Synthesis and characterization of long-CNTs by electrical arc discharge in deionized water and $\mathrm{NaCl}$ solution
}

\author{
Amir Hossein Sari ${ }^{1}\left[\right.$. Arezoo Khazali ${ }^{1} \cdot$ Sara Sadat Parhizgar ${ }^{1}$
}

Received: 8 November 2017 / Accepted: 4 February 2018 / Published online: 14 February 2018

(c) The Author(s) 2018. This article is an open access publication

\begin{abstract}
In this study, electrical arc discharge method is used for the synthesis of multi wall carbon nanotubes (CNTs). The advantages of applied setup for producing CNTs are simplicity, low-cost procedures and avoiding the multistep purification. The experiments were optimized by submerging graphite electrodes inside deionized water and various concentrations of sodium chloride solution. The purpose of this research is to investigate the effect of liquid medium on growth, size and quality of the CNTs structures. The results show that CNTs of $150 \hat{\mathrm{A}} \mu \mathrm{m}$ length or larger with high purity and quality without using catalyst are produced on the cathode surface. Furthermore, the quantity of CNTs is influenced by NaCl concentration. Scanning electron microscopy, Raman spectroscopy and X-ray diffraction technique were used to characterize the results.
\end{abstract}

Keywords CNTs · Arc discharge

\section{Introduction}

Carbon nanotubes (CNTs) are very attractive materials in the fields of medicine and engineering due to their unique electrical, mechanical and elastic properties [1,2]. There are various methods to produce CNTs in sizable quantities [3-5]. Mainly CNTs are produced by arc discharge, laser ablation and chemical vapor deposition methods $[6,7]$. Prof. Iijima for the first time conducted an arc discharge in a chamber with helium gas at atmospheric pressure [8]. Ebbesen et al. reported the production of large amount of CNTs by arc discharge in a reduced inert-gas atmosphere (e.g., Ar, He, etc.) [9]. Using low pressure and noble gasses can be the disadvantages of this method. Ishigami synthesized the CNTs in liquid nitrogen [10]. Liquid environments preferably deny the need for high expenses and complicated equipment. Antisari produced CNTs in a deionized water (DI water) and liquid nitrogen and then compared the results [11]. CNTs produced in both arc discharge and laser ablation methods need to be purified [12]. However, CNTs produced by arc discharge are highly crystalline and have fewer defects than nanotubes produced by other methods [13]. The most

Amir Hossein Sari

sari@ srbiau.ac.ir; amirsari@gmil.com

1 Plasma Physics Research Center, Science and Research Branch, Islamic Azad University, Tehran, Iran common impurities are graphite nanoparticles, amorphous carbon, fullerene, catalytic particles, and metal particles which are either mixed with nanotubes or they are attached on the outer surface. Various methods such as thermal or chemical oxidation, sonication, and annealing can reduce the amount of impurities and amorphous carbon and carbon nanoparticles [14-16]. Purification by thermal method is achieved because the rates of oxidation of CNTs are slower than amorphous carbon, fullerene, and carbon clusters [17].

Important factors for choosing a suitable liquid medium are cooling ability and the minimal evaporation during arc discharge [18]. Although the temperature of liquid nitrogen are very low but the intense evaporation during the arc discharge prevent a proper thermal exchange between the produced substance and its surroundings. Thus, cooling efficiency of liquid nitrogen is less than DI water. In addition, synthesized CNTs show a degraded structure and distorted morphology [19]. On the other hand, due to electrical insulating feature of DI water, the stability of arc discharge is weak.

Therefore, a proper liquid with optimized electrical characteristic, great cooling ability and low vaporization during arc discharge should be found. It is well-known that the cooling power and electrical conductivity of sodium chloride solution is higher than DI water. But the question is: what is the best concentration of $\mathrm{NaCl}$ for production of CNTs. Answering to this question is the main purpose of this paper. 
In this work, the effect of sodium chloride concentration on the growth, size and quality of the structure of CNTs has been investigated.

\section{Experiments}

Figure 1 schematically shows the arc discharge setup in the liquid. The main parts of the facility are: Open-Pyrex vessel, graphite electrodes, copper holders, and DC-high current power supply. The anode electrode (6 $\mathrm{mm}$ in diameter) is connected to the positive pole and it can be moved by a micrometer (see Fig. 1). The cathode (14 mm in diameter) is connected to the positive pole where some part of the product is deposited on top of this electrode. The distance between the electrodes was kept constant at $1 \mathrm{~mm}$. The electrodes were placed parallel to each other and immersed in different concentrations of sodium chloride and DI water solution $(0,0.2,0.25,0.3 \mathrm{M})$. Time durations of 15 and $60 \mathrm{~s}$ for the arc discharge were considered for each concentration. In all experiments the applied current between the electrodes was 90 A. Experimental parameters are shown in Table 1.

After the current flow and producing stable electric arc discharge, the produced materials were: hard crust deposited on the cathode surface, the sedimentary solids in bottom of the vessel, and very small amount of free-floating particles on the surface of the liquid. To purify the samples, the hard crust and the sedimentary solids were put in an oven for $30 \mathrm{~min}$ at $500{ }^{\circ} \mathrm{C}$.

To characterize the produced samples, scanning electron microscopy (SEM, Hitachi SU 3500, $15.0 \mathrm{kV}$ ), Raman spectroscopy (Raman, Takram P50C0R10, Nd:Yag, $532 \mathrm{~nm}$ ) and X-ray diffraction technique (XRD, STADI MP, $\lambda=1.54 \AA$ ) were used.

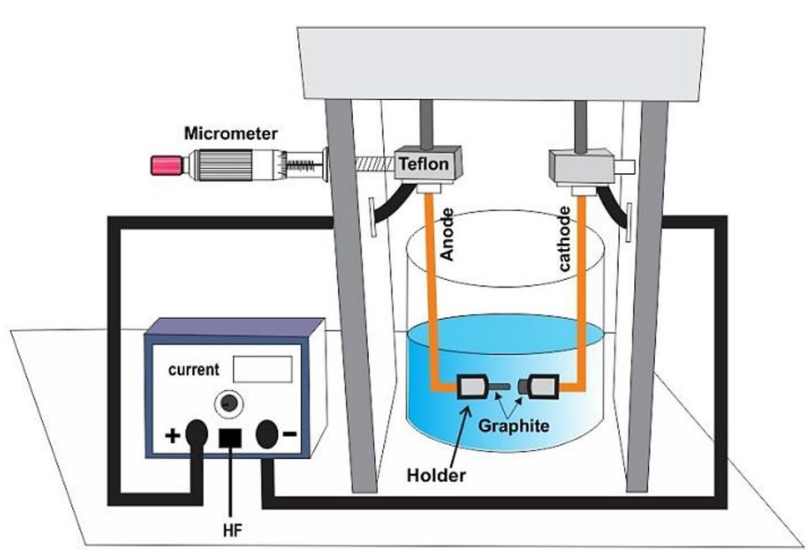

Fig. 1 Schematic view of the arc discharge facility in liquid
Table 1 Experimental parameters

\begin{tabular}{llll}
\hline Sample & $\begin{array}{l}\text { NaCl concentration } \\
(\mathrm{M})\end{array}$ & Time (s) & Current (A) \\
\hline$\# 0$ & 0 & 60 & 90 \\
$\# 1$ & 0.2 & 15 & 90 \\
$\# 2$ & 0.2 & 60 & 90 \\
$\# 3$ & 0.25 & 15 & 90 \\
$\# 4$ & 0.25 & 60 & 90 \\
$\# 5$ & 0.3 & 15 & 90 \\
$\# 6$ & 0.3 & 60 & 90 \\
\hline
\end{tabular}

\section{Results}

Figure 2 shows the SEM image of CNTs synthesized in DI water. High density of nanotubes with rather short length of about 1-2 micrometers is observed in Fig. 2. In Fig. 3 the SEM images of nanotubes at various concentrations of sodium chloride solution $(0.2,0.25$, and $0.3 \mathrm{M})$ for different discharge duration (15 and $60 \mathrm{~s}$ ) are presented. By comparing Figs. 2 and 3, it can be concluded that produced CNTs in $\mathrm{NaCl}$ solution have considerably larger length than in water. The length difference of the synthesized CNTs is from few micrometers in water to about hundreds of micrometers in $\mathrm{NaCl}$ solution. In addition, it confirms that by increasing the $\mathrm{NaCl}$ concentration, the length of CNTs enhances.

Raman spectroscopy is one of the most powerful tools for characterization of carbon nanotubes [19]. In Fig. 4, the results of Raman spectroscopy of produced samples at various concentrations of $\mathrm{NaCl}$ solutions for different discharge duration are demonstrated. It is well-known that CNTs usually reveal peaks at near $156,192,1300$ and $1580 \mathrm{~cm}^{-1}$ in Raman data. For multi wall CNTs, the existing peaks in the

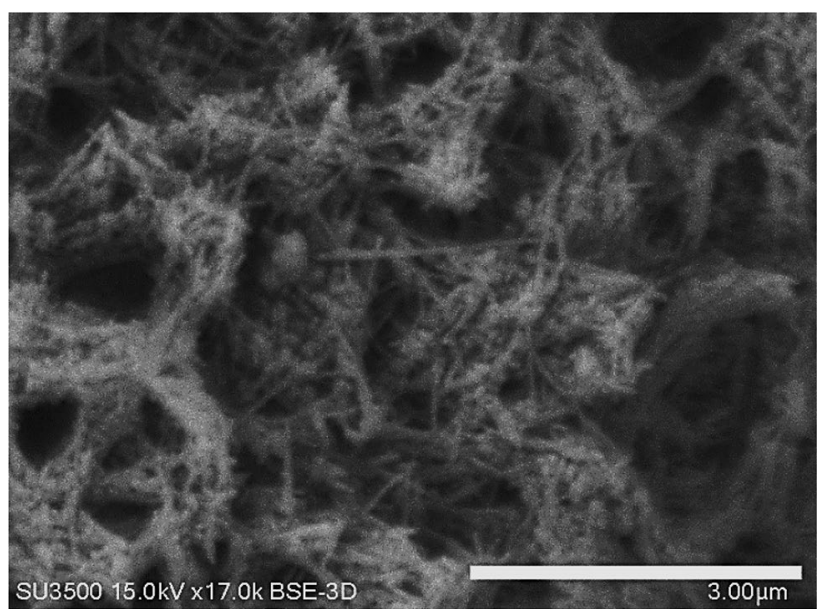

Fig. 2 SEM image of CNTs produced in DI water (sample \#0) 
Fig. 3 SEM images of synthesized CNTs for samples a \#1, b \#2, $\mathbf{c} \# 3, \mathbf{d} \# 4, \mathbf{e} \# 5, \mathbf{f} \# 6$
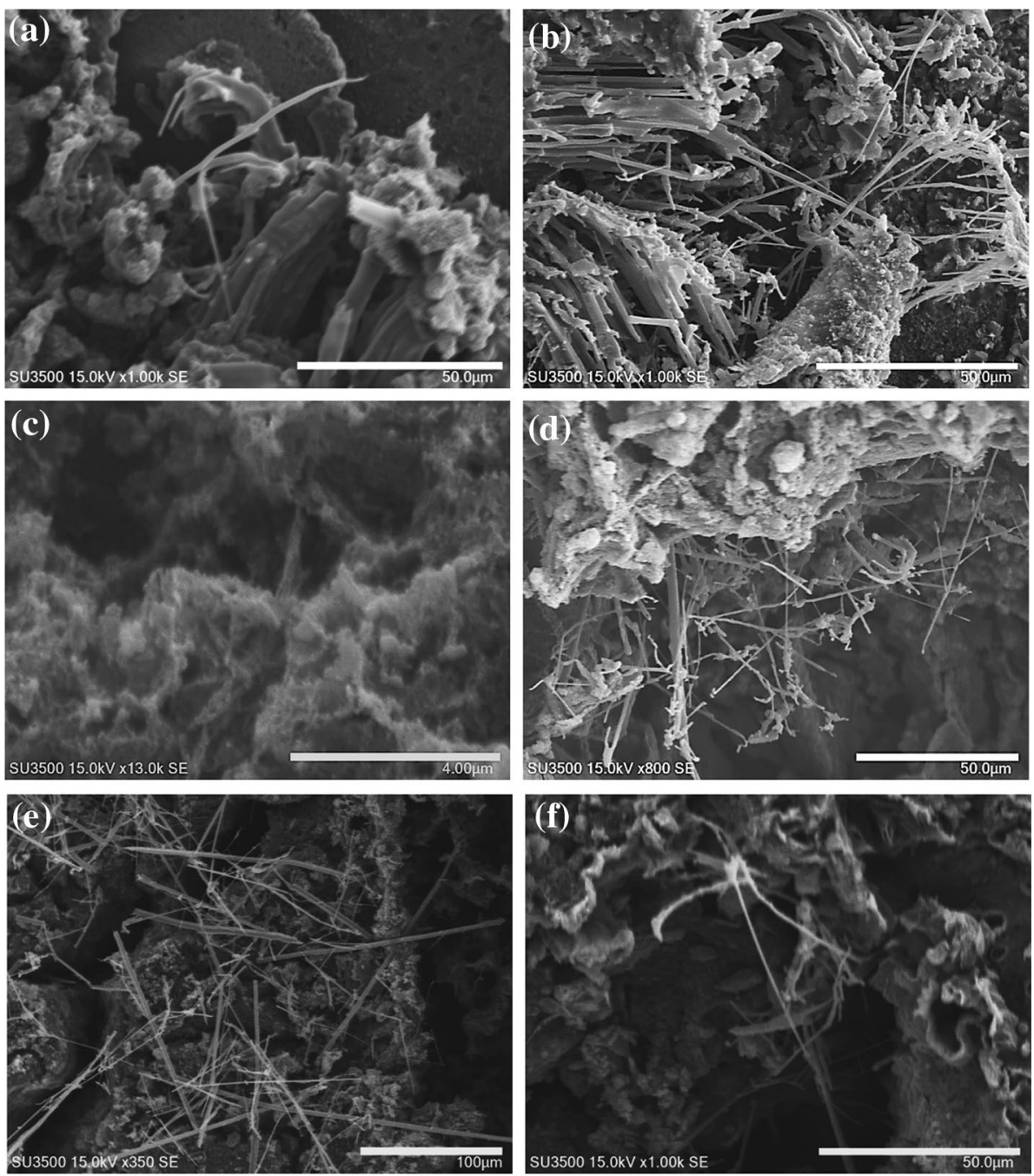

regions $1330-1360,1580-1590$, and $2500-2800 \mathrm{~cm}^{-1}$ are called $D, G$ and $G^{\prime}$ bands, respectively. The strong $G$ peak indicates the presence of graphitized nanotubes and structures [20]. $D$-peak can be an evidence of formation of multi wall CNTs. This is because the curved nature of the graphite sheets enhances the intensity of $D$-peak due to the increase in the electron-phonon coupling [21]. The $G^{\prime}$ peak attribute to one or more layers of graphene or crystalline graphite and its enhancement may be associated to the increase in the growth of graphene multilayer [20].

The ratio of intensity of $G$ band to $D$ band shows the ratio of graphitic structures to amorphous products [22]. Higher ratio of $G$ band to $D$ band is corresponded to the large quantity of CNTs [23]. Similar intensities of these bands indicate a high amount of structural defects [19]. According to Fig. 4, in our case the ratio for samples \#1, \#2, \#3 and \#4 are equal to $2.34,2.7,2.38$, and 3.94, respectively. Therefore, by increasing the discharge time duration, large quantities of nanotubes are obtained. This result is confirmed by SEM analysis (see Fig. 3b, d). In contrast to this, $G-D$ ratio for samples \#5 and \#6 are 3.03 and 1.50, respectively. Thus, it can be concluded that better formations of CNTs at highest $\mathrm{NaCl}$ concentration is achieved for lower discharge time. It may be due to better cooling capability, stability of the discharge and lower evaporation of the liquid at this regime.

Results of X-ray diffraction analysis are shown in Fig. 5. In all samples a strong peak near $2 \theta=26^{\circ}$ are related to (002) planes of hexagonal graphite structure. In some literatures this peak has been related to the formation of multi wall CNTs [20, 24, 25]. As SEM results revealed, CNTs diameter in sample \#2 is greater than samples \#4 and \#5. In addition, D-peak intensity in Raman spectra of samples $\# 4$ and \#5 are greater than sample \#2. This is due to the graphite-curved sheets. Furthermore, intensity of (002) peak for the samples \#4 and \#5 is much higher than the intensity of sample \#2 that shows these samples have more walls with same diameter. The decrease of the interlayer spacing with the increase of diameter of the shells and the inner diameter distribution modify the shape of the (002) peaks. The intensity and width of the (002) peaks are related to the number of 

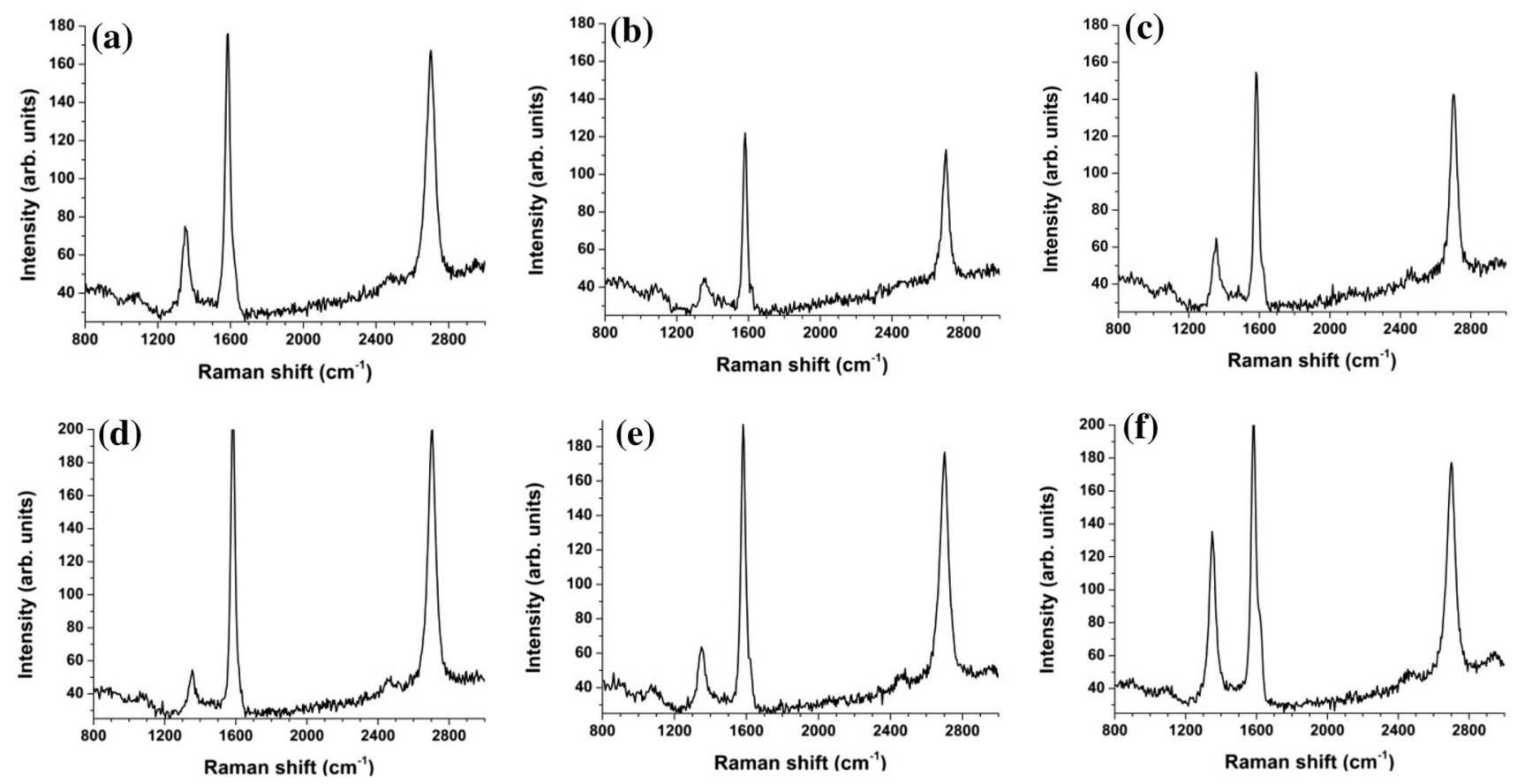

Fig. 4 Raman spectra of produced CNTs for samples a \#1, b \#2, c \#3, d \#4, e \#5, f \#6

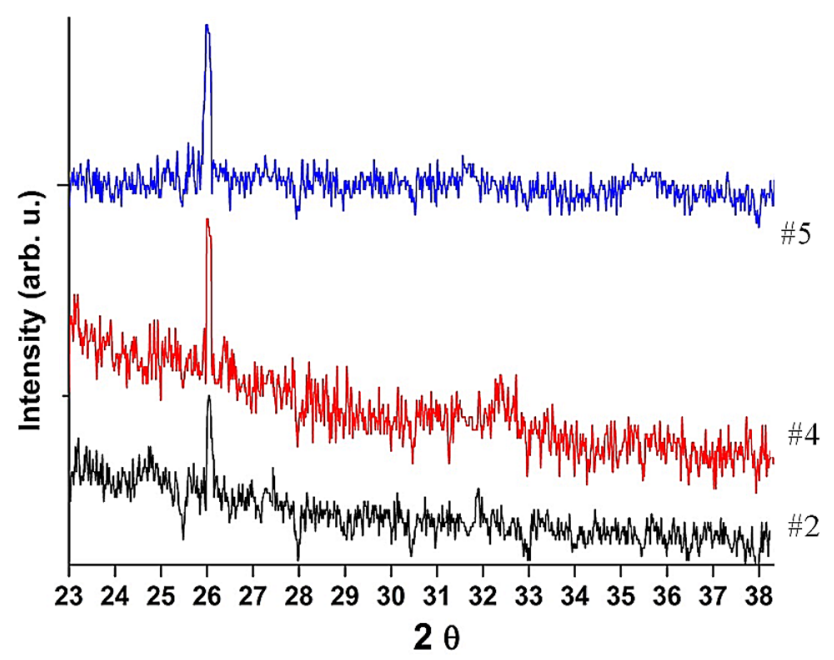

Fig. 5 XRD spectra of CNTs synthesized for samples \#2, \#4, and \#5

layers, the variations of interlayer spacing, the lattice distortions and the carbon nanotube orientation compared to the X-ray incident beam [18].

\section{Conclusion}

In this study, the catalyst-free production of CNTs by arc discharge method in different concentrations of sodium chloride and DI water solution $(\mathrm{NaCl}$ concentration of $0,0.2,0.25$ and $0.3 \mathrm{M}$ ) was performed. SEM results confirmed that with an increase in $\mathrm{NaCl}$ concentration CNTs length grows from few micrometers to more than $150 \hat{\mathrm{A}} \mu \mathrm{m}$. Moreover, quantity of CNTs is influenced by $\mathrm{NaCl}$ concentration. Raman spectroscopy also confirmed the presence of graphite nanotubes and structures. XRD analysis revealed the hexagonal graphite structures with enhanced d-spacing. It can be concluded that arc discharge in liquid is a very cheap method for production of CNTs and the best concentration of sodium chloride solution in DI water is $0.3 \mathrm{M}$ [25].

Open Access This article is distributed under the terms of the Creative Commons Attribution 4.0 International License (http://creativeco mmons.org/licenses/by/4.0/), which permits unrestricted use, distribution, and reproduction in any medium, provided you give appropriate credit to the original author(s) and the source, provide a link to the Creative Commons license, and indicate if changes were made.

\section{References}

1. Paradise, M., Goswami, T.: Carbon nanotubes-production and industrial application. Mater. Des. 28(5), 1477-1489 (2007)

2. Ajayan, P.M., Zhou, O.Z.: Applications of carbon nanotubes. Carbon nanotubes, pp. 391-425. Springer, Berlin (2001)

3. Zheng, L.X., O'Connell, M.J., Doorn, S.K., Liao, X.Z., Zhao, Y.H., Akhadov, E.A., Hoffbauer, M.A., Roop, B.J., Jia, Q.X., Dye, R.C., Peterson, D.E.: Ultralong single-wall carbon nanotubes. Nat. Mater. 3(10), 673-676 (2004) 
4. Prasek, J., Drbohlavova, J., Chomoucka, J., Hubalek, J., Jasek, O., Adam, V., Kizek, R.: Method for carbon nanotubes synthesisreview. J. Mater. Chem. 21(40), 15872-15884 (2011)

5. Ghodselahi, T., Solaymani, S., Akbarzadeh Pasha, M., Vesaghi, M.A.: Ni nanoparticle catalyzed growth of MWCNTs on $\mathrm{Cu}$ Nps@ a-C: H substrate. Eur. Phys. J. D 66(299), 1-5 (2012)

6. Ando, Y., Zhao, X., Sugai, T., Kumar, M.: Growing carbon nanotube. Mater. Today 7(10), 22-29 (2004)

7. Szabó, A., Perri, C., Csató, A., Giordano, G., Vuono, D., Nagy, J.B.: Synthesis methods of carbon nanotubes and related materials. Materials 3(5), 3092-3140 (2010)

8. Iijima, S.: Helical microtubules of graphitic carbon. Nature 354, 56-58 (1991)

9. Ebbesen, T.W., Ajayan, P.M.: Large scale synthesis of carbon nanotubes. Nature 318, 220-222 (1992)

10. Ishigami, M., Cumings, J., Zettl, A., Chen, S.: A simple method for the continuous production of carbon nanotube. Chem. Phys. Lett. 319, 457-459 (2000)

11. Antisari, M.V., Marazzi, R., Krsmanovic, R.: Synthesis of multiwall carbon nanotubes by electric arc discharge in liquid environments. Carbon 41(12), 2393-2401 (2003)

12. Popov, V.N.: Carbon nanotubes: properties and application. Mater. Sci. Eng. R Rep. 43(3), 61-102 (2004)

13. Saravanan, M.S., Babu, S.P., Sivaprasad, K., Jagannatham, M.: Techno-economics of carbon nanotubes produced by open air arc discharge method. Int. J. Eng. Sci. Technol. 2(5), 100-108 (2010)

14. Hou, P.X., Liu, C., Cheng, H.M.: Purification of carbon nanotubes. Carbon 46(15), 2003-2025 (2008)

15. Mathur, R.B., Seth, S., Lal, C., Singh, B.P., Rao, R., Dhami, T.L., Rao, A.M.: Co-synthesis, purification and characterization of single- and multi-walled carbon nanotubes using the electric arc method. Carbon 45, 132-140 (2007)

16. Hou, P., Liu, C., Tong, Y., Xu, S., Liu, M., Cheng, H.: Purification of single-walled carbon nanotubes synthesized by the hydrogen arc-discharge method. J. Mater. Res. 16(9), 2526-2529 (2001)

17. Park, Y.S., Choi, C., Kim, K.S., Chung, D.C., Bae, D.J., An, K.H., Lim, S.C., Zhu, X.Y., Lee, Y.H.: High yield purification of multiwalled carbon nanotubes by selective oxidation during thermal annealing. Carbon 39(5), 655-661 (2001)

18. Wang, S.D., Chang, M.H., Ming-Der Lan, K., Wu, C.C., Cheng, J.J., Chang, H.K.: Synthesis of carbon nanotubes by arc discharge in sodium chloride solution. Carbon 43(8), 1792-1795 (2005)

19. Costa, S., Borowiak-Palen, E., Kruszynska, M., Bachmatiuk, A., Kalenczuk, R.J.: Characterization of carbon nanotubes by Raman spectroscopy. Mater. Sci. Pol. 26(2), 433-441 (2008)

20. Chaudhary, K.T., Bahtti, K.A., Rafique, M.S., Jamil, H., Ali, J., Yupapin, P.P., Saktiot, O., Bidin, N.: Formation of multi-walled carbon nanotubes and graphene in methane arc discharge plasma. Digest J. Nanomater. Biostruct. 9(4), 1297-1308 (2005)

21. Sengupta, J., Jacob, C.: The effect of Fe and Ni catalysts on the growth of multiwalled carbon nanotubes using chemical vapor deposition. J. Nanopart. Res. 12(2), 457-465 (2010)

22. Thomas, B., Epron, F.: Characterization method of carbon nanotubes: a review. Mater. Sci. Eng. B 119(2), 105-118 (2005)

23. Park, Y.S., Kim, K.S., Jeong, H.J., Kim, W.S., Moon, J.M., An, K.H., Bae, D.J., Lee, Y.S., Park, G.S., Lee, Y.H.: Low pressure synthesis of single-walled carbon nanotubes by arc discharge. Synth. Met. 126(2), 245-251 (2002)

24. Singh, D.K., Iyer, P.K., Giri, P.K.: Diameter dependence of interwall separation and strain in multiwalled carbon nanotubes probed by X-ray diffraction and Raman scattering studies. Diam. Relat. Mater. 19(10), 1281-1288 (2010)

25. Sharma, R., Sharma, A.K., Sharma, V.: Synthesis of carbon nanotubes by arc discharge and chemical vapor deposition method with analysis of its morphology, dispersion and functionalization characteristics. Cognet Eng. 2(1), 1094017 (2015). (1-10)

Publisher's Note Springer Nature remains neutral with regard to urisdictional claims in published maps and institutional affiliations. 